\title{
Re-configurable multi-channel high- speed FBG strain sensing system for vibration analysis in oil risers
}

Glynn D. Lloyd, Lorna A. Everall, Kate Sugden, Ian Bennion

Glynn D. Lloyd, Lorna A. Everall, Kate Sugden, Ian Bennion, "Re-configurable multi-channel high-speed FBG strain sensing system for vibration analysis in oil risers," Proc. SPIE 5855, 17th International Conference on Optical Fibre Sensors, (23 May 2005); doi: 10.1117/12.623801

Event: 17th International Conference on Optical Fibre Sensors, 2005, Bruges, Belgium 


\title{
Re-configurable, multi-channel, high-speed FBG strain sensing system for vibration analysis in oil risers
}

\author{
Glynn D. Lloyd ${ }^{* a}$, Lorna A. Everall ${ }^{\mathrm{a}}$, Kate Sugden ${ }^{\mathrm{b}}$, Ian Bennion ${ }^{\mathrm{b}}$ \\ ansensys Limited, 6 \& 7 Compass Point, Hamble, Southampton, Hampshire, SO31 4RA, UK \\ ${ }^{\mathrm{b}}$ Photonics Research Group, Aston University, Aston Triangle, Birmingham, B4 7ET, UK
}

\begin{abstract}
Eight re-configurable, synchronized resonant cavity time-division-multiplexed FBG sensor interrogators provide 16,800 high-resolution measurements-per-second from 280 axially embedded strain sensors, for the analysis of vortexshedding-induced vibration and bending in a composite oil riser pipe.
\end{abstract}

Keywords: Resonant cavity, time-division-multiplexing, FBG strain sensing, vibration analysis

\section{INTRODUCTION}

The use of fiber Bragg gratings (FBGs) for the embedded strain sensing of composite materials is well established and has been the focus of numerous review papers [1,2]. For many 'smart-structures' fewer than twenty strain sensors are needed and the interrogation rate can be just a few Hertz, but some reported systems have employed much higher interrogation frequencies for vibration analysis. In this paper the authors report the development of a multi-channel resonant cavity time-division-multiplexed (TDM) FBG strain measurement system for analysis of the vibration and bending induced in a vertically oriented, composite, oil riser pipe.

Vibration and bending in oil risers results from vortex shedding, which is created by the strong marine currents present in locations where the pipes are deployed. The exact nature of this resonance, and its consequences, are exceedingly complex and so the construction and verification of mathematical models to predict this behavior requires the collection of experimental data.

To collect such data a model riser pipe was manufactured in glass-fiber composite and was fitted with numerous embedded FBG strain sensors. This was then attached to a 'sinker' weight and towed vertically through the sea at a range of controlled velocities to simulate real marine conditions. During this towing the reported strain measurement system was employed in numerous software-selectable interrogation modes to collect extensive strain data. This data was subsequently processed to produce crucial vibration and curvature information for mathematical model verification.

\section{SYSTEM CONFIGURATION}

The modes of oscillation and resonance of a riser pipe can occur both across and in the direction of the dominant marine current, and can also vary considerably in frequency and amplitude, and with depth. Therefore, sensors were required at short intervals along the full length of the pipe and at multiple locations around the circumference. Additionally, due to the high level of induced strain from self-weight and deployment, coupled with the criterion for full vibration and curvature data, each of the sensors was required to deliver absolute strain measurement with high resolution, across a large strain range.

Figure 1 shows the composite pipe and the position of eight separate fiber sensor arrays. Each array contained 35 identical FBG sensors, which were embedded into the model pipe during its manufacture. The fibers were orientated at $45^{\circ}$ intervals around the circumference, such that sensors from each fiber were co-located at equal spacing in the axial direction. The sensors were manufactured in singlemode fiber and were all of $4 \%$ reflectivity, with a nominal zerostrain central wavelength of $1550 \mathrm{~nm}$.

\footnotetext{
*Email: glynn.lloyd@insensys.com; Tel: +44 2380 604300; Fax: +44 2380 604301; Website: www.insensys.com
} 


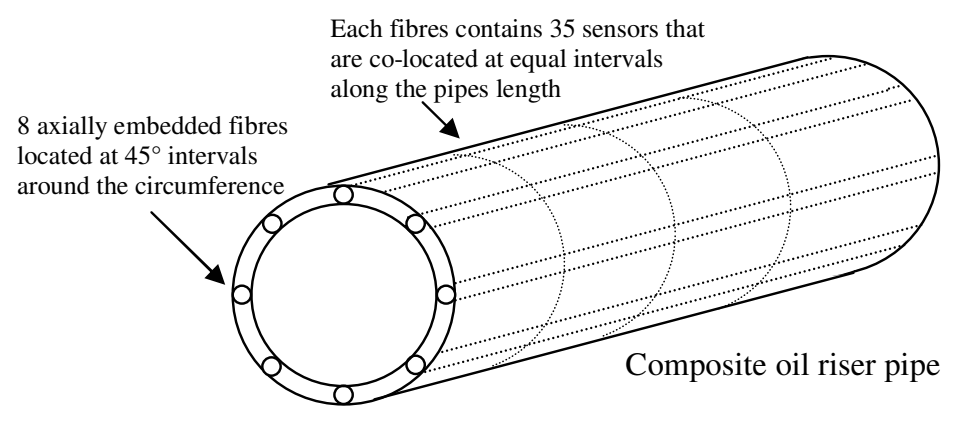

Figure 1. A schematic of the composite riser pipe showing the location of the 280 FBG strain sensors

To accurately resolve the curvature and vibration information into both direction and magnitude it was crucial that the measurement from each sensor was accurately timed and deterministic. In particular, to minimize uncertainty error in the bending analysis, it was highly preferential to provide simultaneous measurement of each set of co-located sensors (i.e. those located at the same position with respect to length along the pipe).

To achieve this criterion with microsecond timing precision each sensor array was connected to a separate resonant cavity TDM interrogator and then all eight interrogators were hardware synchronized to the output signal from a separate synchronizer board. The actual circuit configuration for a single interrogator is shown in Figure 2. This was similar to that presented in our previous work [3,4], but in this instance a diffractive element spectrometer was used in preference to the ratiometric filter. The use of this resonant cavity architecture provided numerous advantages in terms of both performance and cost, which included:

- The ability to interrogate 35 sensors per fiber, with a full 10,000 sensing range for each sensor

- A high sensing resolution per sensor of $<3$, with low signal noise of $<4$ (RMS)

- A very high interrogation rate of 2,100 measurements per second (per interrogator)

The use of identical, low reflectivity 'commodity' sensors also provided considerable cost savings, since this removed the requirement for bespoke array design, allowed the use of through-coating grating fabrication and enabled simpler system deployment.

A further inherent advantage of the resonant cavity TDM interrogation technique was an ability to easily re-configure the sensor configuration, on a per-test basis, to match the changing data requirements of the research; by simple adjustment of software controls on a graphical user interface the sensor configuration could be changed from using a single sensor (per array) at an interrogation rate of $2,100 \mathrm{~Hz}$ per sensor, through to measurement of all 280 sensors at a rate of $60 \mathrm{~Hz}$ per sensor.

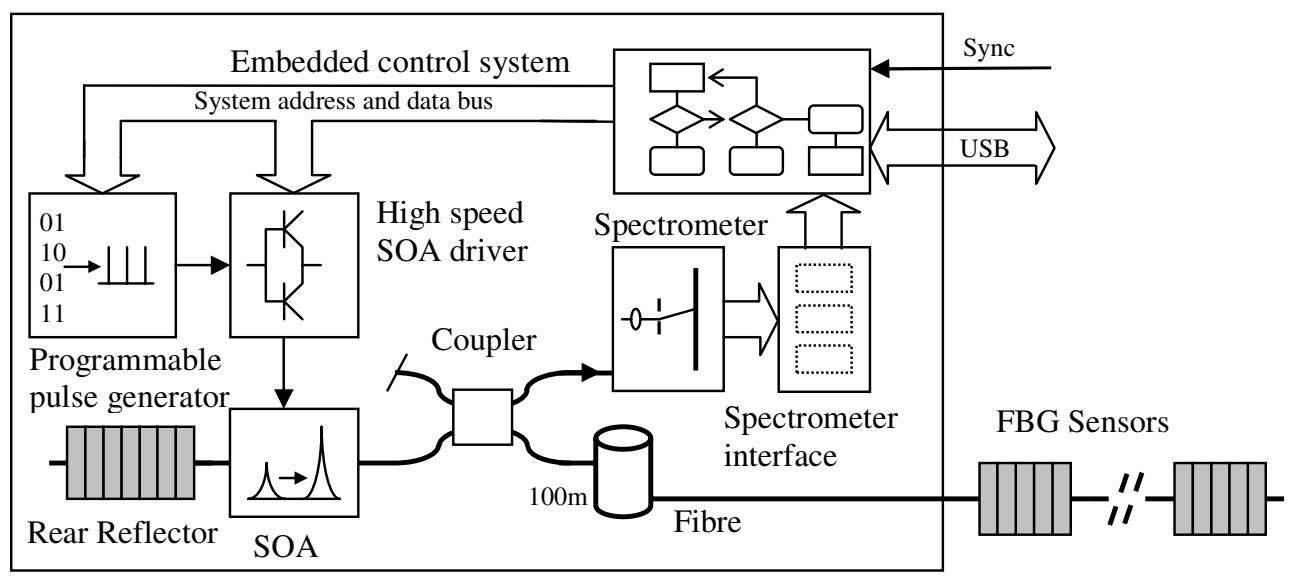

Figure 2. An overview of a single resonant cavity TDM FBG sensor interrogator 
Figure 3 shows the electrical and optical configuration for the complete sensor system. The 8 sensor arrays are shown connected to 8 interrogators, which were then connected in pairs to 4 single board computers (SBCs) via a USB interface. The SBCs were loaded with an embedded Linux operating system and communicated with a Windows-based graphical host PC via a local TCP/IP Ethernet network. Also connected to the network was the hardware synchronizer unit, which was constructed from a low-cost, 8-bit micro-controller.

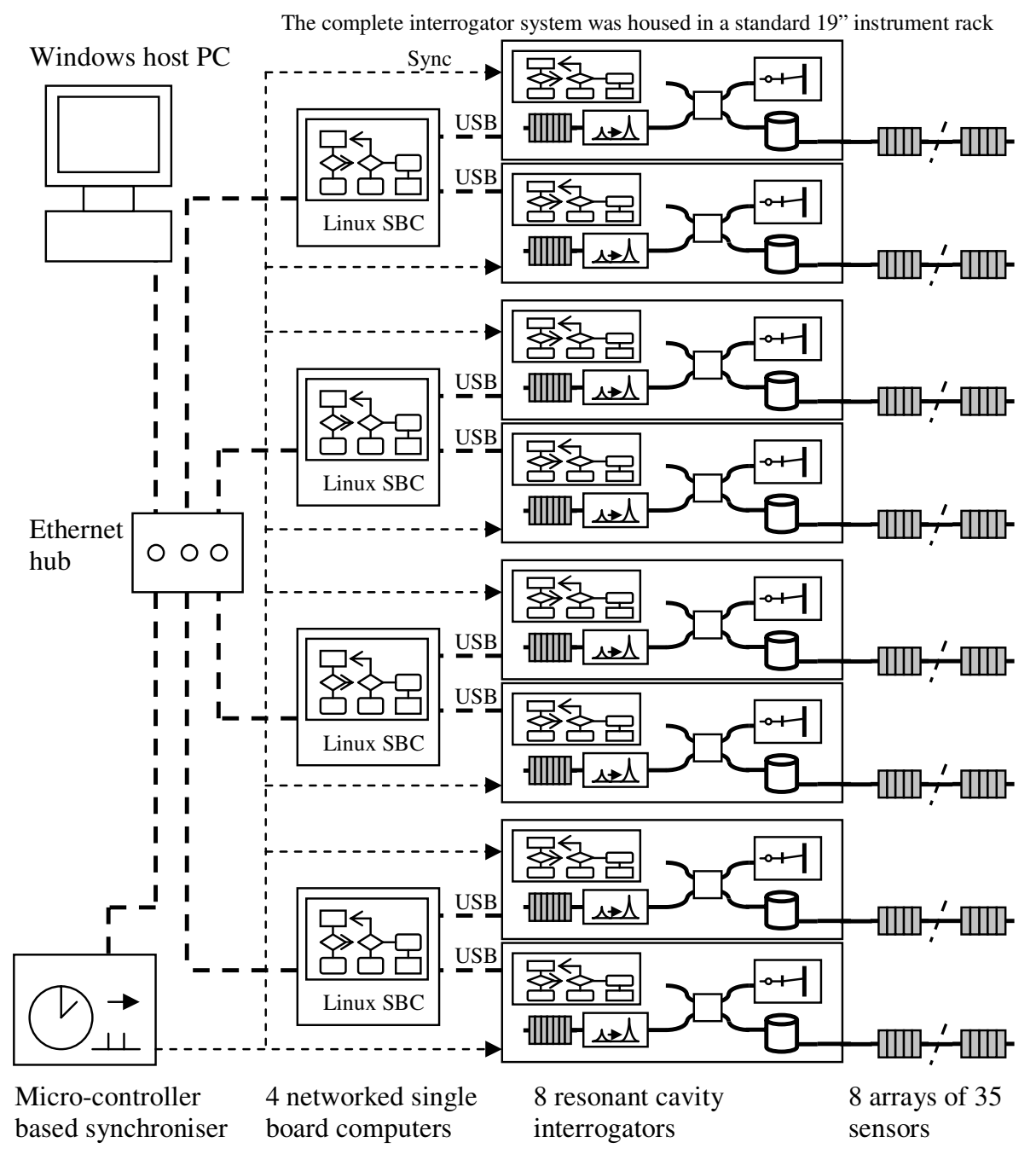

Figure 3. An overview of the complete interrogation system, showing the eight synchronized resonant cavity interrogators, the networked single board computers and the eight FBG arrays (each containing 35 identical, low-reflectivity sensors)

Figure 4 shows an internal and external view of the completed interrogation system, which was mounted into a 19" cabinet enclosure and connected to the model riser pipe using a multi-way waterproof optical connector. 

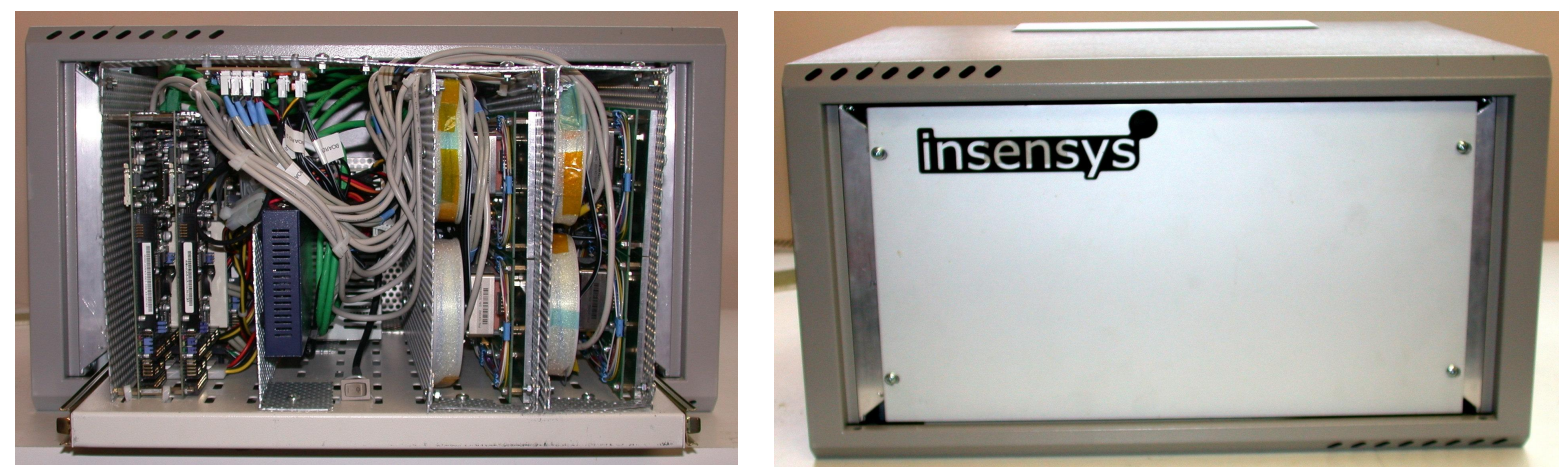

Figure 4. Photographs showing, (a) the internal and (b the external view of the complete interrogation system, when mounted in the 19" rack enclosure

\section{CONCLUSION}

The time-division-multiplexing of 280 identical wavelength, low reflectivity, low-cost FBG sensors using multiple hardware-synchronized resonant cavity interrogators has been used to provide precisely timed, high speed, simultaneous strain measurement across 8 separate fiber channels, for the analysis of vibration and bending in oil riser pipes.

By providing versatile control software and using all-solid-state interrogators that were capable of delivering a combined 16,800 measurements per second, tailoring of the sensor configuration from just eight active sensors interrogated at $2,100 \mathrm{~Hz}$, through to the measurement of all 280 sensors at $60 \mathrm{~Hz}$ each, was possible without any compromise on the 10,000 strain range and micro-strain resolution of each sensor.

\section{ACKNOWLEDGEMENTS}

The authors would like to acknowledge their colleagues at Insensys Limited for their valuable contributions to this work. Glynn Lloyd would like to thank UK EPSRC for partially funding his work.

\section{REFERENCES}

1 A. D. Kersey, M. A. Devis, H. J. Patrick, M. LeBlanc, K. P. Koo, C. G. Askins, M. A. Putnam and E. J. Friebele, "Fiber grating sensors", J. Lightwave Technol., vol.15, 1997, pp.1442-1463

2 K. Lau, L. Yuan, L. Zhou, J. Wu, C. Woo, 'Strain monitoring in FRP laminates and concrete beams using FBG sensors', Composite Structures, vol.51, 2001, pp.9-20

3 G. D. Lloyd, L. A. Everall, K. Sugden and I. Bennion, "Resonant cavity time-division-mulitplexed fibre Bragg grating sensor interrogator", IEEE Photon. Technol. Lett., vol.16, no.10, 2004, pp.2323-2325

4 G. D. Lloyd, L. A. Everall, K. Sugden and I. Bennion, "Resonant cavity based fibre Bragg grating sensor interrogation using ratiometric detection", Opt. Commun. (in press) 